\title{
Quantifying Redundant/Synergistic Interactions Between Cardiorespiratory Reflexes and Cardiac Control Mechanisms During Light-to-Moderate Bicycle Exercise
}

\author{
Alberto Porta ${ }^{1,2}$, Beatrice Cairo ${ }^{1}$, Vlasta Bari ${ }^{2}$, Emanuele Vaini ${ }^{2}$, Beatrice De Maria ${ }^{3}$, \\ Mara Malacarne ${ }^{4,5}$, Massimo Pagani ${ }^{4}$, Daniela Lucini ${ }^{4,5}$ \\ ${ }^{1}$ Department of Biomedical Sciences for Health, University of Milan, Milan, Italy \\ ${ }^{2}$ Department of Cardiothoracic, Vascular Anesthesia and Intensive Care, IRCCS Policlinico San \\ Donato, San Donato Milanese, Milan, Italy \\ ${ }^{3}$ IRCCS Istituti Clinici Scientifici Maugeri, Italy \\ ${ }^{4}$ Dipartimento di Biotecnologie Mediche e Medicina Traslazionale, University of Milan, Milan, Italy \\ ${ }^{5}$ Exercise Medicine Unit, Humanitas Clinical and Research Center, Rozzano, Milan, Italy
}

\begin{abstract}
Some information domain metrics have been devised to quantify whether subsystems contribute to information carried by a target variable more or less than the sum of their separate contributions leading to synergistic or redundant behaviors respectively. We evaluated the net balance between synergy and redundancy of cardiac and cardiorespiratory controls from spontaneous variability of heart period (HP) and respiration (R). HP variability and $R$ were recorded from 16 healthy subjects (22-58 yrs, 6 males) at rest (REST), during bicycle ergometer exercise at $10 \%, 20 \%$ and $30 \%$ of the maximum load and recovery. The net balance between synergy and redundancy was estimated via a linear regression approach along with HP mean, HP variance, HP power in the high frequency (HF, from 0.15 to $0.45 \mathrm{~Hz}$ ) band $\left(H F a_{H P}\right)$ and squared $H P-R$ coherence at $H F\left[K_{H P-}^{2}\right.$ $\left.{ }_{R}(H F)\right]$. HP mean, $H P$ variance and $H F a_{H P}$ power significantly decreased during exercise and $K_{H P-R}^{2}(H F)$ tended to decline. The net balance between synergy and redundancy remained unvaried and was not correlated to traditional variability markers. Despite vagal withdrawal, complex cardiac and cardiorespiratory control interactions are preserved during exercise and indexes of synergy and redundancy might provide extra information that more traditional variability markers cannot capture.
\end{abstract}

\section{Introduction}

The regulation of heart period (HP) is noninvasively quantified by assessing various features of spontaneous HP fluctuations such as the amplitude of HP variability at the respiratory rate [1] or the degree of predictability of the current HP based on previous HP values [2].
Cardiorespiratory reflexes [3], comprising Hering-Breuer reflex [4], Bainbridge reflex [5] and central modulation of cardiac vagal motoneuron activity and responsiveness [6], are a specific subset of the overall HP regulatory mechanisms that act in response to respiration (R). While the assessment of cardiac control is grounded on the analysis of spontaneous HP variations [7], the evaluation of the cardiorespiratory reflexes requires the simultaneous recording of $\mathrm{HP}$ variability and $\mathrm{R}$ as a minimal setup and the use of a causal approach assessing the dynamical interactions from R to HP [8-11]. Due to the specificity of cardiorespiratory reflexes one might be interested in evaluating the degree of synergy/redundancy of cardiorespiratory reflexes and the overall cardiac control mechanisms [10-12]. These markers might provide indexes complementary to those more usually computed in HP variability analysis.

The aim of the study is to quantify the net balance between synergy and redundancy of cardiorespiratory and cardiac controls during a protocol known to alter cardiorespiratory coupling via a graded sympathetic activation, namely incremental light-to-moderate bicycle exercise $[7,13,14]$. Synergy/redundancy was quantified via a linear regression method grounded on the predictive information decomposition approach taking $\mathrm{HP}$ as the target series and exploiting the past of $\mathrm{HP}$ and $\mathrm{R}$ to characterize the cardiac control and cardiorespiratory reflexes respectively [9].

\section{Experimental protocol and analysis}

\subsection{Experimental protocol}

A full description of the experimental protocol and population is reported elsewhere $[7,13,14]$. We studied 16 
healthy humans (aged from 22 to 58 yrs, $40 \pm 12$ yrs, 6 males). We acquired electrocardiogram (ECG) from lead II (ECG Bioamplifier, Marazza, Monza, Italy) and R signal via thoracic belt (Marazza, Monza, Italy). Signals were sampled at $300 \mathrm{~Hz}$. After a period of 10 minutes for stabilization with the subjects in a recumbent position on the bicycle ergometer (Lode, Groningen, The Netherlands), we recorded ECG and R for 10 minutes at rest (REST), for 4 minutes during electronically braked exercise at 10\% (EXE1), 20\% (EXE2) and 30\% (EX33) of the maximum load, and for 10 minutes during recovery (REC). The maximum load was identified individually during a session of incremental exercise until volitional exhaustion carried out the day before. The study was in keeping with the Declaration of Helsinki for medical research involving human subjects and was approved by the ethical review board of the "Luigi Sacco" Hospital, Milan, Italy. Written signed informed consent was obtained from all subjects.

\subsection{Beat-to-beat time series extraction}

The HP was approximated as the temporal distance between two consecutive R-wave peaks detected over the ECG. R signal was downsampled in correspondence of the first R-wave apex of the ECG providing the onset of the $n$th HP. R-wave apexes were fixed using parabolic interpolation. The detections of the R-wave peaks were visually checked and manually corrected in case of erroneous identifications. In case of ectopic beats, the series were corrected by means of linear interpolation. Corrections never exceeded $5 \%$ of the total values analyzed in each session. HP and R series of 256 consecutive values were selected randomly in each experimental session $[9,15]$. We computed mean and variance of HP series indicated, respectively, as $\mu_{\mathrm{HP}}$ and $\sigma_{\mathrm{HP}}^{2}$ and expressed in $\mathrm{ms}$ and $\mathrm{ms}^{2}$.

\subsection{Power spectral analysis}

Power spectral densities of $\mathrm{HP}$ and $\mathrm{R}$ series were computed via an autoregressive power spectral approach. The coefficients of the autoregressive model were identified from $\mathrm{HP}$ and $\mathrm{R}$ series via traditional least squares technique solved via the Levinson-Durbin recursion. The number of coefficients was optimized through the Akaike information criterion. Power spectral density was estimated from the identified coefficients of the autoregressive model and the variance of the prediction error. The power spectral density was decomposed into spectral components. They were classified as high frequency (HF) whether their central frequency dropped into the range from 0.15 to $0.45 \mathrm{~Hz}$
$[9,15]$. The sum of the HF components of HP series, expressed in absolute units (i.e. $\mathrm{ms}^{2}$ ), was labeled as $\mathrm{HFa}_{\mathrm{HP}}$. The $\mathrm{HF}_{\mathrm{a}, \mathrm{HP}}$ power is taken as a marker of respiratory sinus arrhythmia (RSA) representing the visible effect of the action of R on HP variability [1].

\subsection{Squared coherence analysis}

Squared coherence function between $\mathrm{HP}$ and $\mathrm{R}$ series at the $\mathrm{HF}$, termed $\mathrm{K}_{\mathrm{HP}-\mathrm{R}}(\mathrm{HF})$, is traditionally exploited to assess the strength of the cardiorespiratory coupling. It represents the degree of linear correlation between the two series at the respiratory rate. $\mathrm{K}_{\mathrm{HP}-\mathrm{R}}^{2}(\mathrm{HF})$ was calculated by sampling at HF the ratio between the square cross-spectrum modulus between $\mathrm{HP}$ and $\mathrm{R}$ series to the product of their power spectra. $\mathrm{K}_{\mathrm{HP}-\mathrm{R}}^{2}(\mathrm{HF})$ ranged from 1 (full correlation) to 0 (null correlation) and was dimensionless. The estimation of the cross-spectrum was grounded over the identification of a bivariate autoregressive process and the model order was set to 10 [9]. Sampling was performed in correspondence of the weighted central frequency of the $\mathrm{R}$ components in $\mathrm{HF}$ band, where the weights were the powers of the $R$ components [9].

\subsection{Quantification of the net balance between synergy and redundancy}

Synergy between two stochastic variables in relation to a third one is observed when the contribution of the two variables to the information carried by the third variable is greater than the sum of their separate contributions. When the opposite situation is observed redundancy is detected. Let us consider the series $\mathrm{HP}=\left\{\mathrm{HP}_{n}, n=1, \ldots, N\right\}$ and $\mathrm{R}=\left\{\mathrm{R}_{n}, n=1, \ldots, N\right\}$ as two realizations of length $N$ of the stochastic processes $H P$ and $R$. The $H P$ and $R$ processes describe the functioning of cardiac and respiratory systems respectively. Let us assume that the triplet of stochastic variables $H P_{n}, \quad \boldsymbol{H P}_{n-1, p}=\left[H P_{n-1} \cdots H P_{n-p}\right] \quad$ and $\boldsymbol{R}_{n, p+1}=\left[R_{n} R_{n-1} \cdots R_{n-p}\right]$ represents the current state of the cardiac system, the past state of the cardiac system and the past state of the respiratory system. Moreover, let us assume that the action of $\boldsymbol{H P}_{n-1, p}$ on $H P_{n}$ and that of $\boldsymbol{R}_{n, p+1}$ on $H P_{n}$ is owing to cardiac and cardiorespiratory control mechanisms respectively. According to the predictive information decomposition method the net balance between synergy and redundancy of the cardiac and cardiorespiratory controls can be computed as a linear combination of mutual information (MI) terms $[5,12]$ as

$$
\begin{aligned}
& I_{H P, R}^{H P}\left(H P_{n}\right)=M I\left(H P_{n} ; H P_{n-1, p}, \boldsymbol{R}_{n, p+1}\right)+ \\
& -M I\left(H P_{n} ; \boldsymbol{H P}_{n-1, p}\right)-M I\left(H P_{n} ; \boldsymbol{R}_{n, p+1}\right)
\end{aligned}
$$



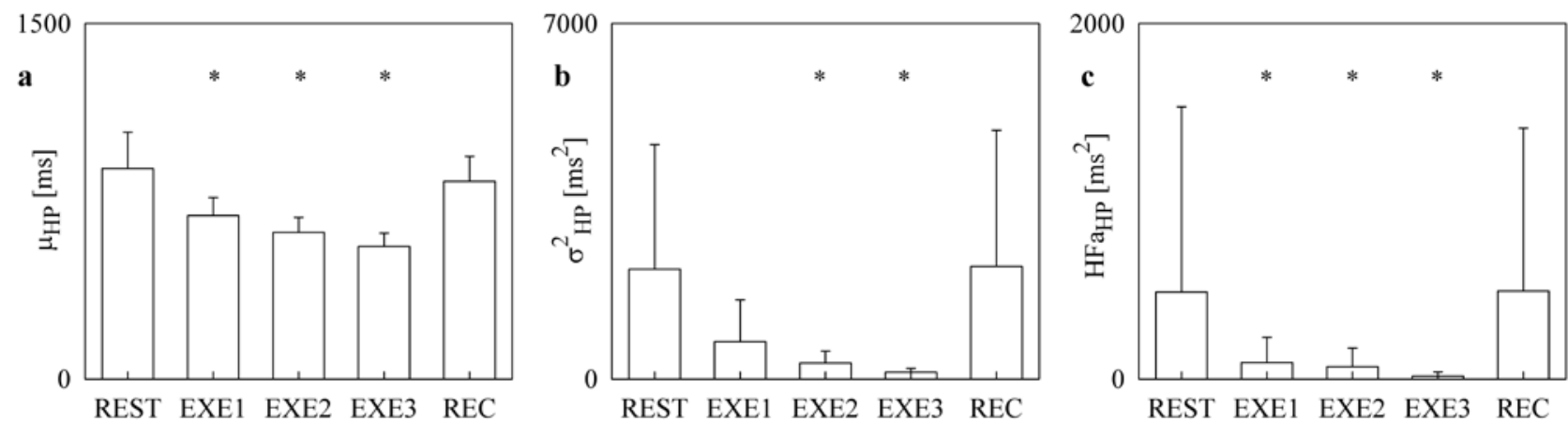

Figure 1. The error bar graphs show $\mu_{\mathrm{HP}}(\mathrm{a}), \sigma_{\mathrm{HP}}^{2}$ (b), and $\mathrm{HFa}_{\mathrm{HP}}$ (c) as a function of the experimental condition (i.e. REST, EXE1, EXE2, EXE3 and REC). The symbol * indicates a significant difference compared to REST with $p<0.05$.

where $M I\left(H P_{n} ; \boldsymbol{H} \boldsymbol{P}_{n-1, p}, \boldsymbol{R}_{n, p+1}\right)$ is the MI between $H P_{n}$ and the vector variables $\boldsymbol{H} \boldsymbol{P}_{n-1, p}$ and $\boldsymbol{R}_{n, p+1}$ when they are jointly considered and $M I\left(H P_{n} ; \boldsymbol{H} \boldsymbol{P}_{n-1, p}\right)$ and $M I\left(H P_{n} ; \boldsymbol{R}_{n, p+1}\right)$ are the MIs between $H P_{n}$ and the vector variables $\boldsymbol{H} \boldsymbol{P}_{n-1, p}$ and $\boldsymbol{R}_{n, p+1}$ when they are separately considered. $I_{H P, R}^{H P}\left(H P_{n}\right)>0$ indicates dominant synergy, while $I_{H P, R}^{H P}\left(H P_{n}\right)<0$ prevalent redundancy. MI estimates were obtained via a linear regression approach solving an identification problem analogous to that of identifying the coefficients of the autoregressive model in Sect.2.3 but accounting for the exogenous effect of $\mathrm{R}$ as well [9].

\subsection{Statistical analysis}

One-way repeated measures analysis of variance (Dunnett's test for multiple comparisons), or Friedman one-way repeated measures analysis of variance on ranks when appropriate (Dunnett's test for multiple comparisons), was applied to test the significance of the changes of indexes versus REST. Pearson correlation analysis was carried out to assess the degree of the correlation of $I_{H P, R}^{H P}\left(H P_{n}\right)$ on traditional variability markers of HP series (i.e. $\mu_{\mathrm{HP}}$ and $\sigma_{\mathrm{HP}}^{2}$ ) and on more usual indexes of cardiorespiratory coupling [i.e. $\mathrm{HFa}_{\mathrm{HP}}$ and $\left.\mathrm{K}_{\mathrm{HP}-\mathrm{R}}^{2}(\mathrm{HF})\right]$. Pearson product moment correlation coefficient $r$ and type I error probability $p$ were calculated. Data were reported as mean \pm standard deviation. A $p<0.05$ was always considered as significant.

\section{Results}

Figure 1 shows $\mu_{\mathrm{HP}}, \sigma_{\mathrm{HP}}^{2}$ and $\mathrm{HFa}_{\mathrm{HP}}$ as a function of the experimental condition (i.e. REST, EXE1, EXE2, EXE3 and REC). The effect of the exercise protocol was evident: indeed, $\mu_{\mathrm{HP}}$ (Fig.1a) and $\mathrm{HFa}_{\mathrm{HP}}$ (fig.1c) decreased compared to REST irrespective of the exercise load, while the decline of $\sigma_{\text {HP }}^{2}$ (Fig.1b) became visible during EXE2 and EXE3.

Figure 2 and Figure 3 have the same structure as Fig.1 but they show $\mathrm{K}_{\mathrm{HP}-\mathrm{R}}^{2}(\mathrm{HF})$ and $I^{H P}{ }_{H P, R}\left(H P_{n}\right)$ respectively. A tendency toward a decrease of $\mathrm{K}_{\mathrm{HP}-\mathrm{R}}^{2}(\mathrm{HF})$ during EXE sessions was manifest but it was not significant due to the dispersion of the indexes (Fig.2). Conversely, $I^{H P}{ }_{H P, R}\left(H P_{n}\right)$ did not exhibit any apparent trend during EXE sessions (Fig.3).

We computed the degree of the correlation of $I^{H P}{ }_{H P, R}\left(H P_{n}\right)$ on $\mu_{H P}, \sigma_{H P}^{2}, H_{H P}$ and $K_{H P-R}^{2}(H F)$. Data were pooled together regardless of the experimental condition. None of the markers was found to be significantly associated with $I_{H P, R}^{H P}\left(H P_{n}\right)$ with $p<0.05$.

\section{Discussion}

We applied a predictive information decomposition approach [9-12] to assess the net balance between synergy and redundancy of cardiac and cardiorespiratory regulations in governing the HP evolution. Cardiac control mechanisms comprise reflexes adjusting HP in response to $\mathrm{R}$ [4-6] as well as regulatory mechanisms targeting again HP but driven by variations of physiological variables different from $\mathrm{R}$ (e.g. arterial pressure) [8-10]. The evaluation was carried out during an experimental protocol known to alter the difference between the minimum and maximum HPs during a respiratory cycle, i.e. the RSA, being the apparent consequence of the cardiorespiratory coupling [1]. While

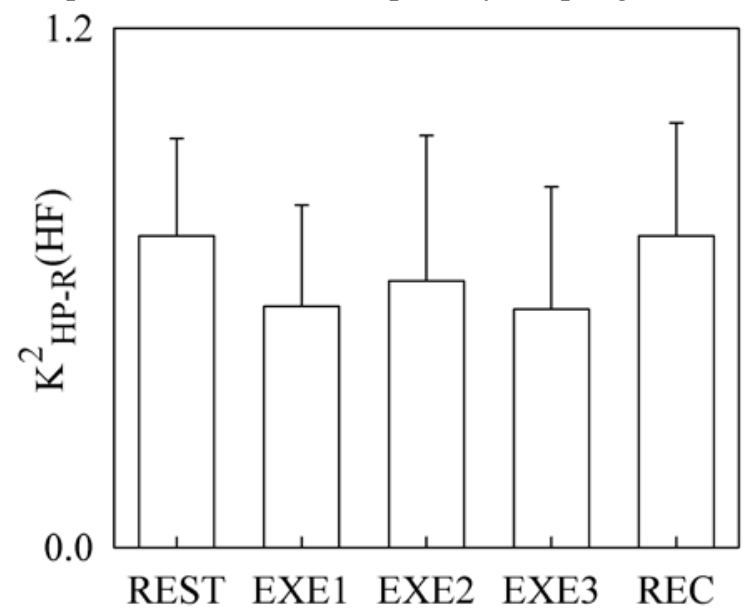

Figure 2. The error bar graph shows $\mathrm{K}_{\mathrm{HP}-\mathrm{R}}^{2}(\mathrm{HF})$ as a function of the experimental condition (i.e. REST, EXE1, EXE2, EXE3 and REC). 


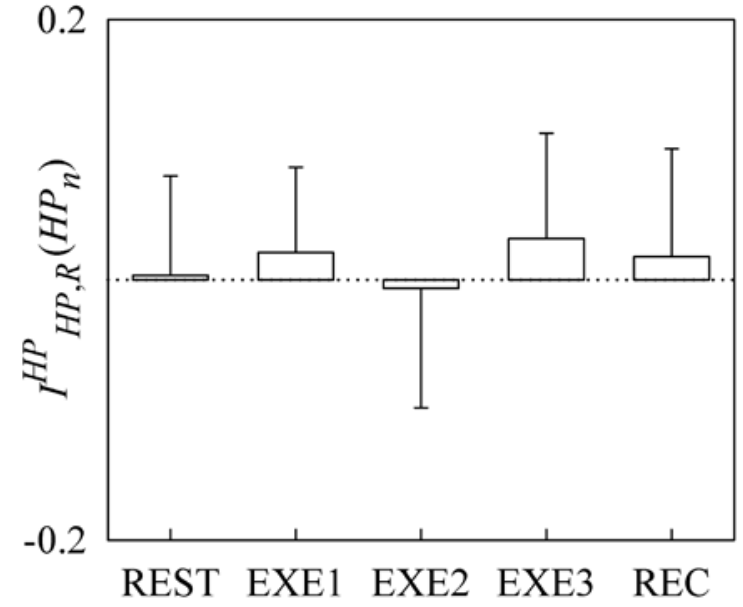

Figure 3. The error bar graph shows $I^{H P}{ }_{H P, R}\left(H P_{n}\right)$ as a function of the experimental condition (i.e. REST, EXE1, EXE2, EXE3 and REC).

bicycle exercise decreased significantly RSA, as estimated via $\mathrm{HFa}_{\mathrm{HP}}$, and tended to decline the strength of the HP-R association, as estimated with $\mathrm{K}^{2}{ }_{\mathrm{HP}-\mathrm{R}}(\mathrm{HF})$, the net balance between synergy and redundancy of cardiac and cardiorespiratory controls remained unvaried. We conclude that a sympathetic activation induced by bicycle exercise $[7,13,14]$ was not able to affect this peculiar feature. This finding is emphasized by the uncorrelation of $I^{H P}{ }_{H P, R}\left(H P_{n}\right)$ with traditional indexes characterizing HP variability and its link to $\mathrm{R}$. This finding is particularly remarkable whether it is compared to our previous observation that controlled breathing at a frequency slower than the spontaneous one in healthy subjects was able to increase $\mathrm{HFa}_{\mathrm{HP}}$ and $\mathrm{K}_{\mathrm{HP}-\mathrm{R}}^{2}(\mathrm{HF})$, raise redundancy and evoke a certain degree of correlation between synergy/redundancy indexes and more traditional cardiorespiratory variability markers [9]. Indeed, experimental protocols evoking opposite modifications of $\mathrm{HFa}_{\mathrm{HP}}$ and $\mathrm{K}_{\mathrm{HP}-\mathrm{R}}^{2}(\mathrm{HF})$ did not produce trivial courses of $I_{H P, R}^{H P}\left(H P_{n}\right)$.

\section{Conclusion}

This study stresses that a marker measuring the net balance between synergy and redundancy of cardiac and cardiorespiratory controls provides complementary information to more classical variability indexes derived from HP series and its interactions with $R$. This complementarity is likely to be linked to the specific experimental protocol evoking sympathetic activation and vagal withdrawal associated to the physical challenge.

\section{References}

[1] B. Pomeranz et al., "Assessment of autonomic function in humans by heart-rate spectral-analysis," Am. J. Physiol., vol. 248, pp. H151-H153, 1985.
[2] A. Porta et al., "Short-term complexity indexes of heart period and systolic arterial pressure variabilities provide complementary information," J. Appl. Physiol., vol. 113: pp. 1810-1820, 2012.

[3] M. Elstad et al., "Cardiorespiratory interactions in humans and animals: rhythms for life," Am. J. Physiol., vol. 315, pp. H6-H17, 2018.

[4] B.H. Taha et al., "Respiratory sinus arrhythmia in humans: An obligatory role for vagal feedback from the lungs," $J$. Appl. Physiol., vol. 78, pp. 638-645, 1995.

[5] G.J. Crystal, and M.R. Salem, "The Bainbridge and the "reverse" Bainbridge reflexes: History, physiology, and clinical relevance," Anesth. Analg., vol. 114, pp. 520-532, 2012.

[6] D.L. Eckberg, "The human respiratory gate," J. Physiol., vol. 548, pp. 339-352, 2003.

[7] A. Porta et al., "On the relevance of computing a local version of sample entropy in cardiovascular control analysis," IEEE Trans. Biomed. Eng., vol. 66, pp. 623-631, 2019.

[8] A. Porta et al., "Model-based assessment of baroreflex and cardiopulmonary couplings during graded head-up tilt," Comput. Biol. Med., vol. 42, pp. 298-305, 2012.

[9] A. Porta et al., "Paced breathing increases the redundancy of cardiorespiratory control in healthy individuals and chronic heart failure patients,” Entropy, vol. 20, Art. no. 949, 2018.

[10] A. Porta et al., "Disentangling cardiovascular control mechanisms during head-down tilt via joint transfer entropy and self-entropy decompositions," Front. Physiol., vol. 6, Art. no. 301, 2015.

[11] A. Porta et al., "Quantifying net synergy/redundancy of spontaneous variability regulation via predictability and transfer entropy decomposition frameworks,” IEEE Trans. Biomed. Eng., vol. 64, pp. 2628-2638, 2017.

[12] A.B. Barrett, "Exploration of synergistic and redundant information sharing in static and dynamical Gaussian systems," Phys. Rev. E, vol. 91, Art. no. 052802, 2015.

[13] D. Lucini et al., "Non-invasive assessment of the changes in static and oscillatory components of peripheral pressure/flow relationships produced by moderate exercise in humans,” J. Hypertens., vol. 15, pp. 1755-1760, 1997.

[14] D. Lucini et al., "Selective reductions of cardiac autonomic responses to light bicycle exercise with aging in healthy humans," Auton. Neurosci.-Basic Clin., vol. 110, pp. 5563, 2004.

[15] Task Force of the European Society of Cardiology and the North American Society of Pacing and Electrophysiology, "Heart rate variability. Standards of measurement, physiological interpretation, and clinical use," Eur. Heart J., vol. 17, pp. 354-381, 1996.

Address for correspondence:

Prof. Alberto Porta, PhD

Università degli Studi di Milano

Dipartimento di Scienze Biomediche per la Salute

IRCCS Policlinico San Donato

Via F. Fellini 4, 20097, San Donato Milanese, Milan, Italy

Tel: +39 02 52774382; email: alberto.porta@unimi.it 